\title{
REPORTS
}

\section{Fostering Informatics Education through Teams Olympiad}

\author{
Nadia AMAROLI ${ }^{1}$, Giorgio AUDRITO², Luigi LAURA ${ }^{3}$ \\ ${ }^{1}$ IIS Aldini Valeriani Sirani, Bologna, Italy \\ ${ }^{2}$ Department of Computer Science, University of Torino, Italy \\ ${ }^{3}$ Department of Computer, Control, and Management Engineering, \\ "Sapienza" University of Roma, Italy \\ e-mail:nadia.amaroli@istruzione.it,giorgio.audrito@unito.it,laura@dis.uniroma1.it
}

\begin{abstract}
Even though the International Olympiad in Informatics directly involves a restricted number of pupils from each country, one of its primary goals is stimulating interest in computer science and information technology over the whole younger segment of the world's population. In several countries, this aim has to be accomplished without an active intervention of the Ministry of Education on school programs, relying on the efforts of small devoted organizations. In this context, promoting the involvement of a large number of school teachers may be as crucial as difficult to achieve. Following a 9-year experience of teams competitions in Italy, recently shared with other European countries, we argue that teams Olympiad may be an effective tool for widening the participation of high-school students and teachers, synergistically cooperating with existing individual competitions. On the one hand, teams contests foster peer education, encouraging talented students to help training fellows. On the other hand, these competitions can be more appealing both for average students, valuing group membership more than personal accomplishments, and most importantly, teachers: team achievements are more recognizably linked with the overall school or teacher performance than solitary excellences, resulting in increased returns rewarding the involved subjects.
\end{abstract}

Keywords: team work, programming contest, olympiads in informatics, peer education, programming training. 


\section{Introduction}

Programming competitions are an effective tool to motivate and engage students in informatics concepts (Dagiene and Futschek, 2010). As such, the role played in the last thirty years by the two arguably most important competitions, i.e., the International Olympiads in Informatics (IOI) and the ACM International Collegiate Programming Contest (ICPC), is widely recognized (Audrito et al., 2012; Bloomfield and Sotomayor, 2016; Combéfis et al., 2017; Combéfis and Wautelet, 2014; Dagienè, 2010; Pavlova and Yanova, 2017; Tsvetkova, 2010).

In this paper we report our experience about the International Informatics Olympiad in Teams (IIOT), a recent competition aimed at filling an important gap in secondary school education: competing as a team of contestants (as in the university-targeted ACM ICPC) as opposed to the individual competition characteristic of the IOI. The IIOT builds over a 9-year experience of teams competitions in Italy and, in its second edition (2018), sees the participation of Italy, Romania, Russia, Bulgaria, Moldavia and Sweden.

During these few years of existence, the IIOT encouraged computational thinking and enhanced individual performances in Italy in a measurable way, confirming generally consensus on teamwork-based educational experiences (Tsay and Brady, 2012). In the specific contest of informatics education, teams contests proved to have a number of recognizable positive effects, allowing for peer education, sense of belonging, connection of talented students, involvement of teachers. The recent worldwide ${ }^{1}$ success of the Bebras contest (Dagienè, 2008; Dagienè, 2010), aimed at promoting Informatics (Computer Science, or Computing) and computational thinking among school students at all ages, can also be seen as a proof of the effectiveness of team contests.

This paper is organized as follows:

- Section 2 details the IIOT characteristics and features, including its history, the competition and organizational structures, and, most importantly, how to join it.

- In Section 3 we discuss issues related to the diffusion of programming contests and their application to the IIOT.

- In Section 4 we report data from a national (Italian) point of view on the effects of teams contests.

- Concluding remarks are addressed in Section 5.

\section{International Informatics Olympiad in Teams (IIOT)}

The IIOT aims at motivating secondary school students to cultivate interest in informatics and coding, while testing and proving their problem solving skills, synergistically alongside the individual Olympiad in Informatics. Furthermore, the teamwork-oriented methodology used by this competition targets the exchanging of knowledge and experi-

${ }^{1}$ Currently, in the Bebras website www. bebras . org are listed 58 participating nations plus six that are planning to install a Bebras contest. 
ence among young people with similar interests and qualifications, through the establishment of personal contacts both within and across countries.

More and more often, the world of work operates in contexts in which working groups are pivotal to carry out projects or activities. Teamwork skills, therefore, are usually a prerequisite for all those who are joining the current labor market: in all workplaces, or nearly so, you need to interface with other people to carry on your own activities. It is thus clear that establishing this capability can improve the performance level and lead to a better work environment.

In this competition, teamwork allows to achieve better results from the team's collective talents, from the members' ability to support each other in difficult times, and from the multiplied creativity that comes from the comparison of ideas. Ultimately, this competition experience translates into personal growth as well as improved individual performances, as we will detail in Section 4. Furthermore, the IIOT fills an age gap in team competitions between the Bebras and the ACM ICPC, being aimed at students in secondary schools.

Section 2.1 summarizes the competition history, while Sections 2.2 and 2.3 present the contest structure and organization respectively. Finally, Section 2.4 details on the possible ways to join the competition.

\subsection{History of the Competition}

Teams competitions in Italy started in 2010 with the Olimpiadi di Informatica a Squadre (OIS) thanks to Giorgeliana Carletto, who had the initial idea and the tireless perseverance to make this dream become a reality. In the first edition, participating teams belonged only to Emilia-Romagna (a region in the north of Italy, where the school of Giorgeliana Carletto is located), they were only seven, and the competition itself was carried out through the Croatian Open Competition in Informatics (COCI $)^{2}$. Nowadays, all of the twenty Italian regions participate into the contest, for a total of more than 400 teams, 2250 athletes and 110 schools involved in the 9th edition.

Starting from school year 2014/15 the contest is held on specifically-designed tasks, which were first written in Italian and later in English (from school year 2016/17) for two main reasons: to promote the international dimension of this competition, and because of the central role of this language in the today scientific community (and computer science in particular).

This first edition of the International Informatics Olympiad in Teams was held in Italy from May 17 to 20, 2017 at the IIS Aldini Valeriani-Sirani of Bologna, with the relevant award ceremony on 20 May 2017 at the presence of the former president of the European Commission and Italian Prime Minister Romano Prodi.

The second edition was held in Piatra Neam, Romania, from May 23 to 28, 2018. A mainly informative web site was built by Italian organization for the International project (http://iio.team), as well as a Facebook page of the event (https://

\footnotetext{
${ }^{2}$ http://hsin.hr/coci
} 
$\mathrm{fb} . \mathrm{me} / \mathrm{i}$ io.team). There is also a website for each national competition: the Italian one is reachable at http://oisquadre.it, whilst the Romanian one is available at http://cni.nt.edu.ro/ioit.

Several personalities and public organizations have supported the project in Italy so far, including AICA, the Olympic Committee of the Olimpiadi Italiane di Informatica (OII), the former president of the European Commission and Italian Prime Minister Romano Prodi, Councilor Patrizio Bianchi of the Emilia-Romagna region, Ing. Romano Volta from Datalogic.

\subsection{Competition Structure}

Following the corresponding rule of the IOI, contestants are students enrolled in a school for secondary education, in the country they are representing, during at least September-December in the year before IIOT and who are not older than 20 on July 1 st of the year of IIOT. Students who are studying abroad may represent the country of their nationality. Each team has to consist of four members and up to two reserves. A team can include at most one awarded contestant of the National Individual Olympiad in Informatics in the previous year. No student exchanges are allowed between teams. Each team is given two PCs, without Internet connection besides the official platform, no translators nor dictionaries.

Each national championship has its own rules that can be different from country to country. Usually, the national championship consists of four preliminary competition rounds and one national final round: one preliminary round per month starting from October till January, with the national finals held in the first half of March. Each team is given a username valid for the whole competition and a different password for each round. Each contest lasts 3 hours (4 for international rounds) and usually consists of 7 problems in English, to be solved in C, C++ or Pascal (other languages such as Java or Python may also be allowed in national contests) without special hardware or software requirements. Contestants may submit written questions through the platform to the Scientific Committee concerning the formulation and interpretation of the problems during each round.

The preliminary rounds are held online, on a national dedicated platform with an automatic evaluator (usually CMS (Maggiolo and Mascellani, 2012; Maggiolo et al., 2014)). The national final competition, on-site, selects its participants according to the total scores obtained in the previous 4 rounds, but its score is not added to the previous ones. In Romania, the ten best-ranked teams are selected for the national finals. In Italy, finalist teams are selected based on their region: each region participates with its best-ranked team, plus the ten best teams nation-wise among the remaining ones.

The first one (or two ${ }^{3}$ ) winning teams of each national final competition will participate in the IIOT. All the involved countries can also participate with one more "special

${ }^{3}$ The host country decides the number of participating teams, depending on the number of participating countries. 
guest team", from the national leader schools: their results will not be listed in the official rank, but they will be awarded.

The reference syllabus of the competitions is that of the IOI, however, most problems require a reduced competence set, such as: arrays, sorting and searching, greedy algorithms, recursion, dynamic programming, trees and basic graph algorithms. Given the limited time available to solve many problems, teamwork and cooperation are necessary for winning: no single individual can outperform a group of four people, working in pairs at a same problem and helping each other on the hardest tasks.

\subsection{Organizational Structure}

Enrollment in the national IIOT project (and in the IIOT itself) is completely free for all schools, both public and private. A school for each country is appointed as the leader school to coordinate all the activities of registration, competitions, administration of problems and awarding of the national winning team, as well as to maintain relationships with leading schools of other countries.

Each country in the IIOT is then represented by a National Committee (NC) that consists of four people: the representative of the Ministry of Education or another appropriate institution, the headmaster and a teacher of the leader school and the scientific coordinator. Together, the national committees of regular members form the International Committee (IC) and are responsible of regulation updates, overall organization, and appointing the International Coordinator who represents the IIOT project.

Meanwhile, the Scientific Committee (SC) of each regular participant country consists of few scientific experts, and has the duty to supervise the preparation and evaluation of tasks for the national contests. The host country SC should prepare tasks for the IIOT together with at least one extra proposal. These tasks will then be presented before the contest to the International Scientific Committee (ISC) consisting of the SC of each member country. The ISC has the right to reject tasks proposed by the host country SC, in which case the extra proposals will be considered as replacements.

The General Assembly (GA) is composed of the national committees of each participating country together with leaders of each participating team ${ }^{4}$ and a president nominated by the host country. The GA will approve the cutoff scores for gold, silver and bronze medals.

\subsection{How to Join the IIOT}

There are few different ways to approach to the IIOT, with increasing degrees of involvement. Firstly, you can unofficially attend contests as a team or individually, to test your ability or get to know the competition: starting from the last year, we host an online

\footnotetext{
${ }^{4}$ Guest countries and teams are represented in the GA (even though not in the IC and SC). The distinction between guest and member countries will be clarified in Section 2.4.
} 
mirror contest for this purpose. You can subscribe to the Italian training platform (Di Luigi et al., 2016) before the contests start at https://training.olinfo.it, and during each round you can solve the problems by submitting your solutions. The official and mirror contests have identical tasks, rules and platforms, with the sole exception of starting times: the mirror contest is held USACO-style, so that you can choose when to start your 3-hour time window during the 24 hours following the start of the official contest. Unofficial ranking will be available as soon as the contest starts, on a separate web page than the official one.

Secondly, few schools from a country might want to experimentally join the official competition, testing themselves and their teams, and trying what attending this project means. As experimented this year with Sweden, we propose to freely host these teams (up to 10 for each country) on the Italian or Romanian official platforms, allowing the winning teams of each country to possibly participate as guest at the following IIOT. In this way, Sweden has held its national finals using the Romanian platform, during its contest timing.

Lastly, you might join the IIOT project officially by hosting your own national competition. In this case, you should contact $\mathrm{us}^{5}$ to organize your participation, set up an online contest management system such as CMS (Maggiolo and Mascellani, 2012; Maggiolo et al., 2014), and the annual host country Scientific Committee will provide you their sets of problems for each of the qualifying contests, which you will be free to use, adapt or discard.

Joining the international project is free of charge and you can choose between two types of collaboration. First, you can be a regular member by giving a declaration of intent on hosting a future IIOT edition. In this case, your National Committee shall sit at the table as peer with other National Committees, and your participation into the International Final will be free of charge (except for travel expenses). Otherwise, you could attend it as guest participant: in this case, you will not need to host future IIOT editions nor take part into the decision tables, while still be allowed to attend the International final with your national teams, upon paying a participation fee decided upon discretion of the host country.

\section{Organizing a Team-Based Informatics Contest}

Successfully organizing a competition is a demanding task, involving countless choices for which a definitive answer may not even be possible. We hereby try to motivate and discuss the issues and solutions we encountered during these few years of experience, hoping they could provide a solid ground for further development and help newly joining countries for the setup of their national competitions.

Section 3.1 discusses the goals that motivated our quest for a better competition, Section 3.2 presents the technical issues encountered and respective solutions adopted, Section 3.3 lists the main organizational issues and solutions.

5 iiot@iio.team 


\subsection{Motivating Goals}

During our Italian experience, the basic motivating goal has always been widening the participation to the highest possible extent, in order to maximize the impact of the project. Towards this aim, we need to cope with the plurality and diversity of subjects that take different roles in the competition. Firstly, students may come from very different schools, with a theoretic or technical focus, and have varying sets of skills and competences. Teachers as well may have various backgrounds: mathematics (strongly represented in Italy), computer science or even electronics. Some of them, which are not acquainted with the practice of information technology, need to be supported by technicians in their school and/or possibly by the organizers themselves. University teachers and students need to be involved into the project as well: to prepare tasks, manage the contest platform, help teachers and technicians during the lab's preparation, and overall helping with the organization of the events.

In order for a competition to be successful, a broad participation must be achieved among all roles, by building a network linking together people united by their similar interests in informatics and problem solving. This bring us to the second goal: providing a fertile environment for building connections between different people joined by their passion, in this way encouraging their positive involvement in the subject.

Meanwhile, we are obviously interested into our students personal growth. For a team-based contest, this process can simultaneously involve social and teamwork skills on one side, technical coding and problem solving skills on the other side. As our milestone goal, we aim to improve the performance of students in individual competitions and coding in general, while encouraging a cooperative attitude instead of a more solitary or "nerd" one: looking at mates with respect and tolerance, not estranged but rather actively immersed into the surrounding environment. Regarding the technical skills, we aim in particular at providing a fruitful training for the individual Olympiad: as contests are held regularly and progressively, students can be lead through a growth path directed at their personal performance in individual contests, learning new skills from teachers, mates and experience.

\subsection{Technical and Scientific Issues}

Several technical and scientific issues have to be addressed in order to met the goals just stated. Firstly, in order to handle online contests with thousands of students simultaneously competing, we had to host the CMS platform on the Google Cloud Platform as locally available servers were failing to deliver an acceptable quality of service. We used a standard machine with 50GB SSD storage for the database and main instance of CMS, together with three preemptible machines devoted to the Contest Web Server, and eight preemptible worker machines with high CPU settings. Virtual worker machines on the cloud proved to have higher variability in execution times than worker processes on 
locally controlled hardware, however, the difference was not so significant to impair the evaluation process.

Another subtle issue with online contests is that of ensuring a fair competition without any form of physical control: to address this issue, we opted for an approach mixing automatic tools and teacher accountability. We handed multi-platform instructions to teachers on how to block Internet access for contest computers, while implementing an automatic connection check in the contest website, which sends alert mails to teachers whenever one of their teams is spotted to be connected to the Internet. After the end of the contest, teachers are queried about their teams who hit a certain threshold number of warnings (if any), and we decide together with them whether to penalize the teams in question. Furthermore, a semi-automatic check for plagiarisms is done based on JPlag, ${ }^{6}$ and plagiarisms found are correspondingly penalized. Finally, we plan to implement in CMS for next year a checker ensuring that submitted source code does not contain pragma instructions and inline assembly, which are against the spirit of the competition and prohibited in other contests such as the ACM ICPC.

The selection and preparation of tasks requires a similarly careful attention than that of the technical framework. In order to engage students with different preparation levels and back-grounds, we attempt to build set of tasks with amusing narratives and widely varying scopes, ranging from extremely easy to technically involved or logically tricky. This requires contests made from several problems: we believe that the chosen number of seven problems is appropriate for this purpose. Even though this number is lower than the average number of problems in the ACM ICPC team contests, the discrepancy is mitigated by the presence of subtasks of various difficulty into which each task is fragmented. For next year, we plan to encourage the participation of younger and less experienced contestants by introducing a progressive syllabus, dividing required competences into three increasing levels: a starting level for newbie programmers, an intermediate level with selected topics from school programs, and an advanced level corresponding to the IOI syllabus. The tasks will then be sorted and explicitly marked according to their syllabus level.

As previously mentioned, the involvement of teachers is crucial for the correct running of the competitions, and even more so for enriching the participation and preparation of students. Since team performances are more easily connected to quality teaching than solitary excellences, teachers are often more motivated to get involved into team contests than into individual competitions. In order to promote the involvement of teachers into the competition, we plan for next year to allow and encourage teachers to submit tasks for consideration into the competitions, while acknowledging the task author in the final competition booklets.

\subsection{Organizational Issues}

Among the organizational issues, the first one we had to face is schools and teams enrollment. In the first editions of the OIS, we managed enrollment manually through for-

\footnotetext{
${ }^{6}$ https://jplag.ipd.kit.edu
} 
mal emails. As the number of participating schools and teams grew, manual enrollment became unfeasible, and we had to switch to an online registration form: a first online form is designed for enrolling schools, and then a second online form is sent to enrolled schools for submitting teams. These forms are advertised to schools across all Italy with the help of the Ministry of Education.

Once the enrollment has taken place, it is crucial to keep active channels of communication between the organization and school referents. With the plethora of communication systems available in the modern world (phone calls, text messages, emails, WhatsApp or Telegram) it is sometimes difficult to reach an agreement on a preferred set of communication methods. At the moment, we are encouraging the usage of email for formal communications, and of WhatsApp for urgent, non-official messages. These channels are used for general information and support, but also to gather the school feedbacks needed to pursue the evolution of the competition. Several ideas arising from valuable feedback have been (or are going to be) implemented, such as: unofficial mirror contests, cooperative Internet access control, progressive syllabus.

Besides the communication problems, other issues arise in the practical organization of the national final and international contest. Firstly, computer labs with substantial capacity need to be found: about 60-70 PCs in the national round, 20-30 in the international one (currently). So far, we alleviated part of the technical burden by hosting online even the on-site competitions, reducing needs for specific hardware or configurations, and thus being able to host the competitions in sufficiently large schools (IIS Aldini Valeriani Sirani, Bologna).

Secondly, accommodation and cultural activities need to be prepared for the about 150 people ( 50 for the international contest) participating in the event. In order to increase the reach of the event, we also have to look for VIPs to attend the award ceremony, set up entertainment and a buffet, contact press agencies and media. All of this activities come at a cost, so that funding is usually the most critical issue. There is no magic recipe for fund-raising, and so far we obtained the most from long-term low-budget sponsoring from local businesses, integrated with limited institutional funding. For the upcoming years, we hope to score sponsorships from larger international companies as well (Google and/or Microsoft).

\section{Impact of the Teams Olympiad in Italy}

During these years of teams Olympiad in Italy we collected several positive feedbacks. First of all, we obtained positive reactions from students and teachers resulting in a so-far increasing number of schools and students participating in the teams Olympiad, shown in Fig. 1. The major spike in the graph corresponds to the year 2015 when the teams Olympiad were first disconnected from the COCI and popularized through all Italy. Despite this increasing trend, the number of schools and students participating is still much lower than that of the individual Olympiad: 2250 versus 14531 participants and 110 versus 565 schools in 2018 (below 20\% in both cases). Thus, there is still leeway for a further future increase. 


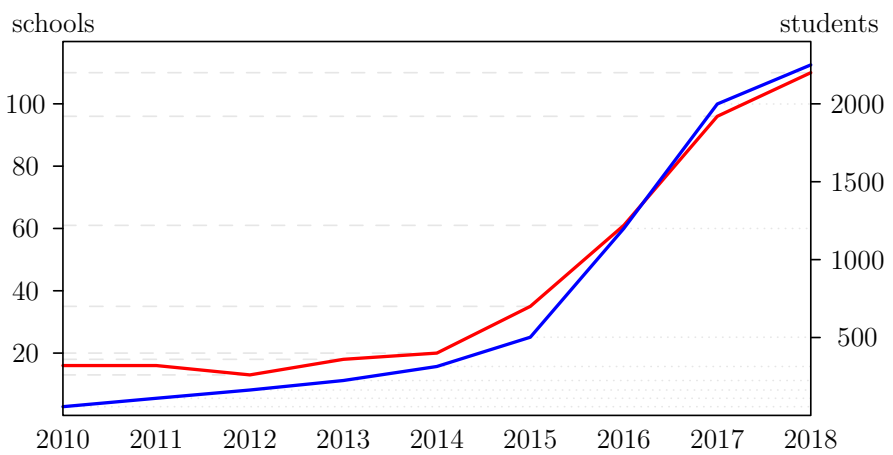

Fig. 1. Schools (red) and students (blue) participating in the Italian teams Olympiad.

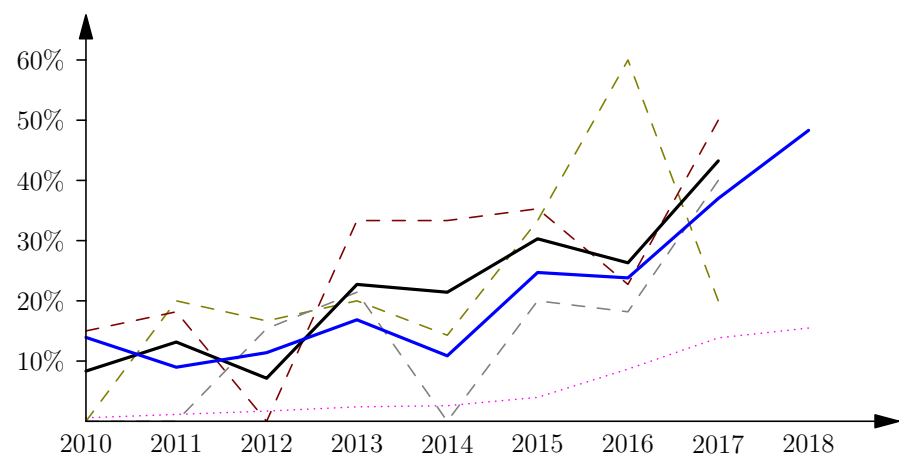

Fig. 2. Percentage of teams Olympiad contestants among individual contestants (dotted magenta), among national finalists of the individual Olympiad (blue), and among medalists in the national contest (dashed bronze, silver, gold, and solid black overall).

As trainers for the individual Olympiad, we also tried to test the effectiveness of the team Olympiads as a form of training and involvement into the subject. In fact, participation in the teams Olympiad highly correlates with better performances at the individual contests, as shown in Fig. 2: a small initial number of students (1\% to $15 \%$ during the years ${ }^{7}$ ) scores a large part of the available places and medals at the individual Italian national contest (about $10 \%$ to $40 \%$ during the years), quite consistently among the different score levels (non medalists, bronze, silver and gold medals). This correlation is due to two cooperating factors: that better students are more eager to participate to new contests, and that students get better by participating into the teams Olympiad.

Among these factors, we claim that the second plays the more relevant role: students get better by practicing with the teams Olympiad. Two main observations substantiate this claim. Firstly, students first participate in the teams Olympiad and then

${ }^{7}$ Data about 2018 medalists is not available as the individual finals are yet to happen at the time of this writing. 
score medals in individual contests more often than medalists happen to join the teams Olympiad. In fact, the regulations forbade medalist to join teams until last year, so that all the data depicted in Fig. 2 regarded no medalists joining the teams Olympiad. Secondly, the overall level of the Italian individual competition is getting better over the last few years.

This increase in the competition level is not only acknowledged by trainers, but also visible in the international results of the Italian team at the IOI (see Fig. 3), where a consistent improvement has taken place in the last few years reaching an all-time best result in 2017 (as average quantile). As a further benchmark of the competition level, we compared the individual informatics Olympiad with the individual mathematics Olympiad. Since 2012, the best students in the Italian mathematical Olympiad are encouraged to join the informatics Olympiad with a special selection process, resulting in a few students being invited to the national contest. Assuming the level of the Italian mathematical Olympiad is approximately constant, we nonetheless observed that a decreasing number of people from this alternate selection is able to reach the training camps (see Fig. 4), suggesting that the level of the main selection is improving.

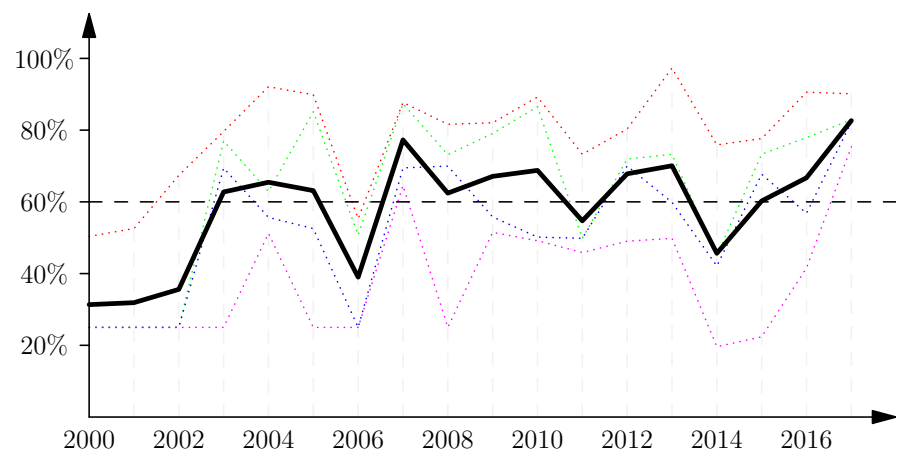

Fig. 3. Individual (dotted) and average (solid black) results of the Italian IOI teams as quantiles (i.e., position on scoreboard as percentage), as reported in http://stats.ioinformatics.org. Missing data for old non-medalists is approximated to the average $25 \%$ quantile. Performance of the four athletes is colored magenta, blue, green, red from 4th to 1 st ranked respectively.

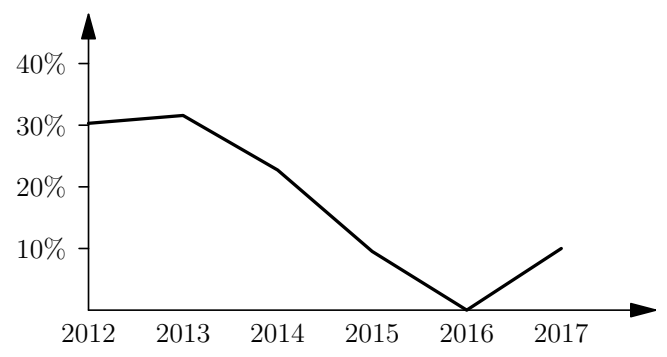

Fig. 4. Percentage of mathematical Olympiad contestants among Italian finalists. 
As the effectiveness of teamwork in education is widely accepted and acknowledged (Tsay and Brady, 2012), we find these results unsurprising. In fact, the specific teamwork promoted by the teams Olympiad can have additional benefits than teamwork in a generic setting. In most Italian high schools, gifted students are evenly distributed across classes, so that they usually do not know each other. Teams Olympiad thus provide a unique setting connecting talented students in the same school, enabling not only peer education but also a sense of belonging that encourages students to pursue their passions and goals.

\section{Conclusions}

In conclusion, we claim that the Olympiad in Informatics in Teams, besides allowing students involved to learn essential Informatics, English and teamwork skills, can significantly improve the students' results for the IOI. In our experience, working together with a team and comparing your own ideas with mates can improve critical thinking, suggest new approaches and solution methods, and promote passion for the subject. Moreover, during its four online contests before the national one, the teams Olympiad helps students during their preparation for the individual Olympiad through problems with bit by bit growing difficulties, engaging them with easier problems and gradually driving them to more complex topics. These claims are corroborated by data on the Italian competitions in the last decade, which shows not only a correlation between participation in teams and overall results, but also an overall positive impact on the competition level. As a consequence, a future expansion of this competition to more countries might be able to positively effect the IOI in a significant way. Towards this aim, we propose four ways to join the teams competition: individually through the mirror online contests, experimentally by relying on the Italian or Romanian organization for the technical side, and regularly both as guest (non-voting, non-hosting) or as member country. We hope these parallel paths will help in engaging an increasing number of students and countries, by allowing anyone to join according to the level of involvement he is able to ensure.

\section{Acknowledgements}

We thank Giorgeliana Carletto for starting both the Italian Informatics Olympiad in Teams (OIS) first and IIOT later, but mostly for providing us an example of what a single individual can achieve when supported by passion and devotion for a greater goal. We also thank all the teachers that believed in the IIOT project, both at a national and international scale, and helped us to spread the diffusion of computer science and computational thinking. Last but not least, we are thankful to Gabriele Farina, Edoardo Morassutto, Luca Chiodini, and William Di Luigi, that managed to make everything work during the contests. 


\section{References}

Audrito, G., Demo, G.B., Giovannetti, E. (2012). The role of contests in changing informatics education: A local view. Olympiads in Informatics, 6.

Bloomfield, A., Sotomayor, B. (2016). A programming contest strategy guide. In: Proceedings of the 47th ACM Technical Symposium on Computing Science Education, SIGCSE'16. New York, NY, USA. ACM, $609-614$.

Combéfis, S., Barry, S.A., Crappe, M., David, M., de Moffarts, G., Hachez, H., Kessels, J. (2017). Learning and teaching algorithm design and optimisation using contests tasks. Olympiads in Informatics, 11.

Combéfis, S., Wautelet, J. (2014). Programming trainings and informatics teaching through online contests. Olympiads in Informatics, 8.

Dagiene, V. (2008). The bebras contest on informatics and computer literacy-students drive to science education. In: Joint Open and Working IFIP Conference. ICT and Learning for the Net Generation, Kuala Lumpur. 214-223.

Dagienè, V. (2010). Sustaining informatics education by contests. In: International Conference on Informatics in Secondary Schools-Evolution and Perspectives. Springer, 1-12.

Dagienė, V., Futschek, G. (2010). Introducing informatics concepts through a contest. In: IFIP Working Conference: New Developments in ICT and Education. Universite de Picardie Jules Verne, Amiens.

Di Luigi, W., Farina, G., Laura, L., Nanni, U., Temperini, M., Versari, L. (2016). oii-web: an interactive online programming contest training system. Olympiads in Informatics, 10, 195-205.

Maggiolo, S., Mascellani, G. (2012). Introducing cms: a contest management system. Olympiads in Informatics, 6, 86-99.

Maggiolo, S., Mascellani, G., Wehrstedt, L. (2014). Cms: a growing grading system. Olympiads in Informatics, 123.

Pavlova, O., Yanova, E. (2017). Olympiads in informatics as a mechanism of training world-class professionals in ict. Olympiads in Informatics, 11.

Tsay, M., Brady, M. (2012). A case study of cooperative learning and communication pedagogy: Does working in teams make a difference? Journal of the Scholarship of Teaching and Learning, 10(2),78-89.

Tsvetkova, M.S. (2010). The olympiads in informatics as a part of the state program of school informatization in russia. Olympiads in Informatics, 4.

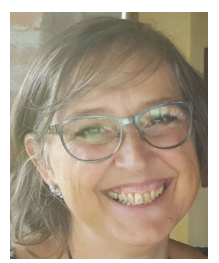

N. Amaroli is a secondary school teacher in computer science labs and a regional school office trainer for digital competences and teaching with innovation technologies. She is the International Coordinator of the IIOT and the deputy president of the OIS. She is the author of the computer science section in the online interactive course for secondary school students, organized by Emilia-Romagna regional school office and by Italian Ministry of Education. 


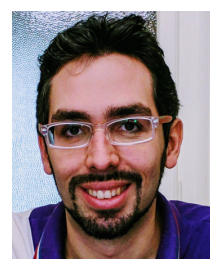

G. Audrito is involved in the training of the Italian team for the IOI since 2006, and since 2013 is the team leader of the Italian team. Since 2014 he has been coordinating the scientific preparation of the OIS and of the first edition of the IIOT. He got a Ph.D. in Mathematics in the University of Turin, and currently teaches "Object Oriented Programming" in the "Piemonte Orientale" University and works as research assistant in the University of Turin.

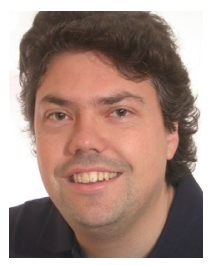

L. Laura is involved in the training of the Italian team for the IOI since 2007, and since 2012 is in the organizing committee of the Italian Olympiads in Informatics. He got a Ph.D. in Computer Science in the "Sapienza" University of Rome, and currently teaches "Webbased Systems Design" in the Tor Vergata University of Rome and "Information Systems" and "Machine Learning" in the LUISS University of Rome. 